\title{
Neutron Structure Functions
}

\author{
J. Arrington, F. Coester, R. J. Holt, T.-S. H. Lee
}

Physics Division, Argonne National Laboratory, Argonne, Illinois 60439, USA

\begin{abstract}
.
Neutron structure functions can be extracted from proton and deuteron data and a representation of the deuteron structure. This procedure does not require DIS approximations or quark structure assumptions. We find that the results depend critically on properly accounting for the $Q^{2}$ dependence of proton and deuteron data. We interpolate the data to fixed $Q^{2}$, and extract the ratio of neutron to proton structure functions. The extracted ratio decreases with increasing $x$, up to $x \approx 0.9$, while there are no data available to constrain the behavior at larger $x$.
\end{abstract}

PACS numbers: $13.60 \mathrm{Hb}, 14.20 \mathrm{Dh}, 21.45 .+\mathrm{v}$ 


\section{Introduction}

Traditionally, much of our information regarding the quark structure of the nucleon has been gleaned from charged lepton scattering. If the one-photon exchange approximation is sufficient, then inclusive structure functions of a target nucleon or nucleus are well defined invariant functions of the cross sections. The structure functions are dimensionless invariant functions of the target four-momentum $P$ and the four-momentum transfer $q$. Thus nucleon structure functions are functions of two invariants, e.g. the momentum transfer, $Q^{2}=-q^{2}$, and the energy transfer, $\nu=P \cdot q / m$, where $m=\sqrt{P^{2}}$ is the nucleon mass. The structure functions can also be taken as functions of other invariants, e.g. $Q^{2}$ and $x$, where $x=Q^{2} / 2 P \cdot q$. It follows from the definition of $x$ that

$$
x=1-\frac{(P+q)^{2}-P^{2}}{2 P \cdot q} \leq 1,
$$

independent of any structure assumptions. For "deep-inelastic" scattering, where

$$
\frac{\nu^{2}}{Q^{2}} \equiv \frac{(P \cdot q)^{2}}{m^{2} Q^{2}} \equiv \frac{Q^{2}}{4 m^{2} x^{2}}>>1,
$$

the parton model implies that the structure functions depend only on $x$ ("scaling"), with a weak logarithmic dependence on $Q^{2}$ ("scaling violations") due to QCD evolution of the parton distributions.

Since the discovery of scaling in deep inelastic electron-proton scattering [1, 2, 3, 4, 5, there have been a number of measurements [6, 7, 8, 9, 10, of the proton structure function. The relation of the proton structure function to the quark structure of the nucleon is model dependent. At medium energies and large $x$, e.g. $Q^{2}=10 \mathrm{GeV}^{2}$ and $x=0.9$, yielding $Q^{2} / 4 m^{2} x^{2}=3.5$, the limit of (2) is not satisfied and the scattering is not "deep inelastic".

To test models of the quark structure of nucleons it is important to have experimental results for both protons and neutrons. The nucleon structure functions for $x>0.4$ are particularly important for constraining models. Since neutron targets are not practical, experimental neutron structure functions must be extracted from deuteron and proton scattering data, without any quark assumptions. This requires consistent Poincaré covariant representations of both the deuteron states and the current-density operators.

Realistic nucleon-nucleon potentials are derived from Lagrangians which determine two-nucleon Green functions, and the interaction dependent exchange currents [11, 12]. Different truncations, regularizations and Lorentz invariant constraints are used for the construction of effective potentials. Parameters are adjusted to accurately fit both deuteron properties and nucleon-nucleon scattering data. These potentials define two-nucleon rest-energy operators (mass operators), which are both Lorentz invariant and Galilean invariant. The difference between the binding energy $M_{d}-2 m$ and $\left(M_{d}^{2}-4 m^{2}\right) / 4 m$ is a negligible relativistic effect. Spin operators are invariant under Galilean boosts, and undergo Wigner rotations under Lorentz boosts. This difference is the source of important relativistic effects. It does not, however, affect inclusive structure functions of unpolarized targets. If these mass operators fit the same scattering and bound state data then they are related by unitary transformations. Such unitary transformations modify the separation of current density operators into one- and two-body operators. 
Given the dynamics specified by an invariant mass operator, unitary representations of the full Poincaré group obtain readily by specification of the generators as functions of the mass operator. The generators of a kinematic subgroup are independent of the mass operator. This choice of kinematics which affects the representation of the current density operators, can be exploited to simplify the relations of deuteron structure functions to nucleon structure functions. The identification of single-nucleon currents is representation dependent. With null-plane kinematics [13] the representations of Lorentz transformations that leave a null vector $n=\{1,-\hat{n}\}$ invariant are independent of the dynamics and only the momentum component $P^{-}=\ell \cdot P, \quad \ell=\{1, \hat{n}\}$ depends on the mass operator. The null vector $n$ can be chosen such that $Q^{+}=n \cdot q=0$. Thus $Q^{2}=Q_{\perp}^{2}-Q^{+} Q^{-}=Q_{\perp}^{2}$ is independent of the dynamics. Kinematic Lorentz transformations which change $P_{\perp}$ do not affect $Q^{+}=0$. With this form of kinematics the impulse assumption for the relevant components of the current density is consistent with current conservation and Lorentz covariance.

This form of kinematics permits a Lorentz invariant convolution relation of the nucleon and deuteron structure functions, which does not require the deep-inelastic approximation, and is independent of the mass spectrum of the final state. Such "smearing" is $Q^{2}$ dependent but converges to the familiar $Q^{2}$ independent convolution when $Q^{2} / \nu^{2}$ is negligible. It is convenient to choose $P^{+}=2 m$ and $P_{\perp}$ such that

$$
P \cdot q=-\vec{P}_{\perp} \cdot \vec{Q}_{\perp}, \quad \nu=-\frac{\vec{P}_{\perp} \cdot \vec{Q}_{\perp}}{m} .
$$

The values of invariants are, of course, independent of such a choice.

In this paper we extract the neutron structure function from proton and deuteron data taking the impulse assumption and an effective two-nucleon mass operator. The main focus is on the consequences of systematic uncertainties and the $Q^{2}$ dependence in the proton and deuteron data. A systematic exploration of the uncertainties associated with using different models of deuteron structure is beyond the scope of this paper.

\section{Cross Sections and Inclusive Structure Functions}

For inclusive electron scattering from an unpolarized target nucleus the Lorentz invariant inclusive cross section can, in the one-photon exchange approximation, be expressed in the form

$$
\frac{d \sigma}{d \Omega d E^{\prime}}=\frac{4 \alpha^{2} E^{\prime 2} \cos ^{2} \theta / 2}{Q^{4} M} F^{\mu \nu}(P, q) f_{\mu \nu},
$$

where $M=\sqrt{P^{2}}$ is the target mass and the electron current tensor,

$$
f_{\mu \nu}=\frac{1}{2}\left(\frac{k_{\mu} k_{\nu}^{\prime}+k_{\mu}^{\prime} k_{\nu}}{\left(k \cdot k^{\prime}\right)}-g_{\mu \nu}\right),
$$

is a bilinear function of the initial and final electron momenta $k$ and $k^{\prime}$. It follows from Lorentz covariance and current conservation that the current tensor $F^{\mu \nu}(P, q)$ is a function of two invariant functions, $F_{1}\left(\nu, Q^{2}\right)$ and $F_{2}\left(\nu, Q^{2}\right)$,

$$
F^{\mu \nu}(P, q)=\left(\frac{q^{\mu} q^{\nu}}{q^{2}}-g^{\mu \nu}\right) F_{1}\left(\nu, Q^{2}\right)+\frac{\tilde{P}^{\mu} \tilde{P}^{\nu}}{P \cdot q} F_{2}\left(\nu, Q^{2}\right),
$$

where

$$
\tilde{P}=P-\frac{P \cdot q}{q^{2}} q
$$


In the frame specified by (3) and $Q_{\perp}=\left\{\sqrt{Q^{2}}, 0\right\}$, the invariant functions are proportional to single components $F^{22}$ and $F^{++}=n_{\mu} n_{\nu} F^{\mu \nu}$ of the current tensor

$$
F_{1}\left(\nu, Q^{2}\right)=F^{22}(P, q), \quad F_{2}\left(\nu, Q^{2}\right)=(P \cdot q) \frac{F^{++}(P, q)}{P^{+^{2}}} .
$$

Since $q^{\mu} f_{\mu \nu}=0$ it follows from these definitions that

$$
\frac{d \sigma}{d \Omega d E^{\prime}}=\frac{4 \alpha^{2} E^{\prime 2} M \cos ^{2} \theta / 2}{Q^{4}(P \cdot q)}\left[F_{2}\left(\nu, Q^{2}\right)+\frac{2 P \cdot q \tan ^{2} \theta / 2}{M^{2}} F_{1}\left(\nu, Q^{2}\right)\right],
$$

and with the ratio of longitudinal and transverse cross sections $R=\sigma_{L} / \sigma_{T}$,

$$
\begin{aligned}
& 1+R\left(\nu, Q^{2}\right)=\frac{M^{2}}{P \cdot q} \frac{F_{2}\left(\nu, Q^{2}\right)}{F_{1}\left(\nu, Q^{2}\right)}\left(1+\frac{\nu^{2}}{Q^{2}}\right), \\
& \frac{d \sigma}{d \Omega d E^{\prime}}=\frac{4 \alpha^{2} E^{\prime 2} M \cos ^{2} \theta / 2}{Q^{4} P \cdot q} F_{2}\left[1+\frac{2\left(Q^{2}+\nu^{2}\right)}{(1+R) Q^{2}} \tan ^{2} \theta / 2\right] .
\end{aligned}
$$

The relation of structure functions to cross sections does not involve any assumptions about the target structure.

\section{Proton and Deuteron Data}

To extract neutron structure functions using convolution relations we need proton and deuteron structure functions at a fixed value of $Q^{2}$. To minimize systematic uncertainties, we use a fit to the proton structure function, $F_{2 p}$, from a recent global analysis and direct measurements of the ratios of deuteron to proton structure function, $R_{d p}=F_{2 d} / F_{2 p}$. Therefore, only the ratios need to be interpolated to fixed values of $Q^{2}$, minimizing the size of these correction. In addition, we minimize sensitivity to normalization uncertainties between data sets by using only measurement of $F_{2 d}$ and $F_{2 p}$ taken from the same experiment when forming the deuteron-to-proton ratios.

For $F_{2 p}\left(x, Q^{2}\right)$, we use the fit from [14, evaluated at $Q_{0}^{2}=12 \mathrm{GeV}^{2}$, which provides parameterizations for $F_{1 p}$ and $F_{2 p}$. The parameters are fit to a large body of data $7,8,15,16,17,18$. The $Q^{2}$ dependence comes from the fit to the data, rather than from a specific model of scaling violations. We compared the fit to the $F_{2 p}$ extraction from several SLAC measurements [16, 19] and BCDMS [8]. For both experiments, the data are taken from the analysis of Whitlow [16, 19], where a relative normalization factor between different experiments is determined and all data are extracted using a common parameterization for $R=\sigma_{L} / \sigma_{T}$. The fit is consistent with the data within the quoted uncertainties, yielding $\chi_{\nu}^{2}=0.86$ when compared to all data for $6<Q^{2}<40 \mathrm{GeV}^{2}$.

For the ratio $R_{d p}\left(x, Q^{2}\right)$, we take the measurements from SLAC [16], BCDMS [8, 20, and NMC [7]. The SLAC data are from a reanalysis of several experiments, and included a fit to the relative normalizations for the different SLAC experiments. While the SLAC, BCDMS, and NMC used different models for $R=\sigma_{L} / \sigma_{T}$, this has no impact on the structure function ratios, as all of the extractions used identical values for $R$ is the proton and deuteron, as suggested by world's data 10 , 18.

In combining all of the $R_{d p}\left(x, Q^{2}\right)$ measurements, we have to account for both statistical uncertainties and the relative normalization of the data sets. The 
uncertainties are broken up into statistical, correlated systematic, and normalization uncertainties. We take the statistical uncertainties to be the combination of the counting statistics and the uncorrelated systematic uncertainties. The correlated systematic uncertainties come mainly from the relative normalization of the different data sets, which yields uncertainties that are typically highly correlated between neighboring $x$ points. The detailed analysis [19] yielded a $1 \%$ overall normalization unceratinty, with smaller relative normalization uncertainties. In combining the results with the BCDMS and SLAC measurements, we also fit a relative normalization offset between the experiments. In the final analysis, we scale the SLAC ratios down by $0.8 \%$, and the BCDMS and NMC results up by $1.9 \%$ and $0.1 \%$ respectively, in all cases within the systematic uncertainties quoted for the ratios. We take a $1 \%$ overall normalization uncertainty on the ratio, and use uncertainties in the relative normalizations of $0.5 \%$ for NMC, $1.0 \%$ for BCDMS, and $0.4-1.1 \%$ for the SLAC experiments, as determined in Ref. [19.

Because the proton structure function parameterization does not include a contribution associated with elastic scattering, the contribution from quasielastic scattering in the deuteron would not be reproduced. The kinematics are chosen such that this contribution is extremely small. A fit to quasielastic scattering data, made to estimate quasielastic and inelastic contributions in the analysis of large $x$ inclusive scattering data 21, indicates that the quasielastic contribution to the $R_{d p}$ ratio is negligible, with a contribution that is at most $0.3 \%$ (for the largest $x$ values), and is always much less than the experimental uncertainties.

There are several points in the results of [19] where the quoted values for $F_{2 d}$ and $F_{2 p}$ do not match the value given for the ratio $F_{2 d} / F_{2 p}$. This usually corresponds to cases where the $x$ or $Q^{2}$ values of the hydrogen and deuterium data did not match exactly, and corrections had to be applied to form ratios at identical kinematics. This correction can be large, especially at large $x$ where $F_{2 p}$ drops rapidly with increasing $x$. This correction is model-dependent, and can introduce a significant uncertainty if the correction is large. In addition, there are a few cases where the discrepancy is too large to be explained by the interpolation (up to $25 \%$ in one case), suggesting an error in either the quoted structure functions or the ratio. To avoid introducing possible bias due to such errors and to minimize the model-dependent corrections associated with large interpolations, we eliminated points where the quoted value for the ratio is more than $2 \%$ from the value calculated from the quoted $F_{2 d}$ and $F_{2 p}$ values. The impact of this cut is negligible except at very large $x$, where the effect is still small compared to the experimental uncertainties. As the final result is insensitive to the exact value of the cut, we do not include any additional uncertainty associated with this cut.

This procedure gives us a large number of individual values of the ratio $R_{d p}\left(x, Q^{2}\right)$, which need to be interpolated to a fixed $Q^{2}$ value, $Q_{0}^{2}$, and binned in $x$. We choose $Q_{0}^{2}=12 \mathrm{GeV}^{2}$, as this is the average $Q^{2}$ value of the large- $x$ data, and limit the overall $Q^{2}$ range so that the average $Q^{2}$ in a given bin is within a factor of two of $Q_{0}^{2}$, to minimize the size of the interpolation correction.

It has been observed that the $Q^{2}$ dependences of the proton and nuclear structure functions [22, 23, 24, 25] and structure function ratios [26] are much smaller when taking fixed values of $\xi=2 x /\left(1+\sqrt{1+4 m^{2} x^{2} / Q^{2}}\right)$ rather than fixed $x$. Thus, we fit the $Q^{2}$ dependence at fixed $\xi$ over the full $Q^{2}$ range of the data, from 3$230 \mathrm{GeV}^{2}$, to extract the $Q^{2}$ dependence. We find $d R_{d p} / d\left(\ln \left(Q^{2}\right)\right) \approx-0.013$, with little $x$ dependence, except for $x \lesssim 0.1$ and $x \gtrsim 0.7$, where the $Q^{2}$ dependence appears 
to be smaller, but is not well measured. This $Q^{2}$ dependence is used to interpolate the individual data points to $Q_{0}^{2}$.

For the final analysis, we use only data with $W^{2}>3 \mathrm{GeV}^{2}$ and $6<Q^{2}<40 \mathrm{GeV}^{2}$, yielding a maximum interpolation correction of $<1 \%$, and an RMS correction of $0.4 \%$. In the regions where the $Q^{2}$-dependence is not well measured, this correction is small compared to the uncertainties, and the associated uncertainty is negligible. After all of the cuts, we have $450 R_{d p}$ measurements, all corrected to $Q_{0}^{2}$. We then bin the data in $x$ to extract a set of points for $R_{d p}\left(x, Q_{0}^{2}\right)$. We combine the data points by taking the statistics-weighted average of the individual points, and the final systematic uncertainty is taken to be the statistics-weighted average of the individual systematic uncertainties.

\section{Nuclear Target Structure}

The current tensor $F^{\mu \nu}$ of the target,

$$
F^{\mu \nu}(P, q)=\operatorname{Tr}\left(\mathcal{F}^{\mu \nu}(q) \rho_{P}\right), \quad \mathcal{F}^{\mu \nu}(q)=I^{\mu}(0) \tilde{I}^{\nu}(q),
$$

is a functional of the current-density operator

$$
I^{\mu}(x)=e^{\imath P \cdot x} I^{\mu}(0) e^{-\imath P \cdot x}, \quad \tilde{I}^{\nu}(q)=\frac{1}{(2 \pi)^{4}} \int d^{4} q e^{-\imath q \cdot x} I^{\mu}(x),
$$

and the target density of a nucleus with spin $j$

$$
\rho_{P}=\frac{1}{2 j+1} \sum_{\sigma=-j}^{+j}|\sigma, P\rangle\langle P, \sigma| .
$$

Lorentz transformations, $\Lambda$, are represented by unitary operators $U(\Lambda)$. The current density operator and the target density transform according to

$$
\begin{aligned}
& U^{\dagger}(\Lambda) \mathcal{F}^{\mu \nu}(q) U(\Lambda)=\Lambda^{\mu}{ }_{\mu^{\prime}} \Lambda^{\nu}{ }_{\nu^{\prime}} \mathcal{F}^{\mu^{\prime} \nu^{\prime}}\left(\Lambda^{-1} q\right), \\
& U^{\dagger}(\Lambda) \rho_{P} U(\Lambda)=\rho_{\Lambda P} .
\end{aligned}
$$

The wave function representing the target state which generates that density may or may not be "manifestly" covariant. If the covariance is not "manifest" there always exist transformations to "manifestly" covariant representations, but there is no substantial significance to "manifest" covariance. Representations of target structure that emphasize the importance of "manifest" covariance intend to emphasize features of quantum field theory which affect the representation of the target structure.

Assuming the only constituents of the deuteron are a proton and a neutron, the deuteron states $|\sigma, P\rangle$ may be represented by functions $\Psi_{\mathbf{P}, M_{d}, \sigma}\left(\mathbf{p}_{p}, \lambda_{p}, \mathbf{p}_{n}, \lambda_{n}\right)$, of the null-plane momenta and the null-plane spins. The null-plane spins are invariant under the kinematic Lorentz transformations that leave the null-plane invariant. These functions are eigenfunctions of an invariant mass operator, $\mathcal{M}$ and the four-momentum operator $P$ :

$$
P_{\perp}=p_{p \perp}+p_{n \perp}, \quad P^{+}=p_{p}^{+}+p_{n}^{+}, \quad P^{-}=\frac{\mathcal{M}^{2}+P_{\perp}^{2}}{P^{+}} .
$$

The associated representation of the current tensor operator, $\left\langle\mathbf{p}_{n}^{\prime}, \lambda_{n}^{\prime}, \mathbf{p}_{p}^{\prime}, \lambda_{p}^{\prime}\left|\mathcal{F}^{\alpha \alpha}(q)\right| \mathbf{p}_{p}, \lambda_{p}, \mathbf{p}_{n}, \lambda_{n}\right\rangle$, is the sum of the impulse term,

$$
\begin{aligned}
& \left\langle\mathbf{p}_{n}^{\prime}, \lambda_{n}^{\prime}, \mathbf{p}_{p}^{\prime}, \lambda_{p}^{\prime}\left|\mathcal{F}_{i m p}^{\alpha \alpha}(q)\right| \mathbf{p}_{p}, \lambda_{p}, \mathbf{p}_{n}, \lambda_{n}\right\rangle \\
& =\delta\left(\mathbf{p}_{n}^{\prime}-\mathbf{p}_{n}\right) \delta_{\lambda_{n}^{\prime}, \lambda_{n}}\left\langle\mathbf{p}_{p}^{\prime}, \lambda_{p}^{\prime}\left|\mathcal{F}_{p}^{\alpha \alpha}(q)\right| \mathbf{p}_{p}, \lambda_{p}\right\rangle
\end{aligned}
$$




$$
+\delta\left(\mathbf{p}_{p}^{\prime}-\mathbf{p}_{p}\right) \delta_{\lambda_{p}^{\prime}, \lambda_{p}}\left\langle\mathbf{p}_{n}^{\prime}, \lambda_{n}^{\prime}\left|\mathcal{F}_{n}^{\alpha \alpha}(q)\right| \mathbf{p}_{n}, \lambda_{n}\right\rangle
$$

and the remainder, $\mathcal{F}_{\text {int }}^{\alpha \alpha}(q)$, which vanishes for large separation of the nucleons. The impulse term is essential for the relation of nucleon and deuteron structure functions. The representation of $\mathcal{F}_{i n t}^{\alpha \alpha}(q)$ is clearly model dependent. Any unitary transformation of $\rho_{p}$ which does not affect the N-N data [27] modifies $\mathcal{F}_{\text {int }}^{\alpha \alpha}(q)$ and thus any conclusion relating nucleon and deuteron structure functions. For $\alpha=+$ and 2, the impulse assumption $\mathcal{F}_{i n t}^{\alpha \alpha}=0$ is consistent with the requirements of Poincaré covariance and current conservation.

It follows from the relation (8) and the impulse assumption that the deuteron structure function is related to the proton and neutron structure functions,

$$
\begin{aligned}
& F_{2 d}\left(\nu, Q^{2}\right)=\frac{1}{2}\left(\bar{F}_{2 p}\left(\nu, Q^{2}\right)+\bar{F}_{2 n}\left(\nu, Q^{2}\right)\right), \\
& \bar{F}_{2 N}\left(\nu, Q^{2}\right)=\int \frac{d p^{+}}{p^{+}} \int d^{2} p_{\perp} \frac{p^{+^{2}}}{P^{+^{2}}} \rho_{P}(\mathbf{p}) \frac{\nu}{\nu_{N}} F_{2 N}\left(\nu_{N}, Q^{2}\right),
\end{aligned}
$$

where $\nu_{N}=-\left(\vec{p}_{\perp} \cdot \vec{Q}\right) / m$. No deep inelastic approximations are involved and there are no target mass effects. Target mass effects are artifacts of inconvenient representations of the deuteron states and the current density operators 28 .

Nucleon momentum densities $\rho_{P}(\mathbf{p})$ obtained from a deuteron wave function satisfy the normalization condition

$$
\int \frac{d p^{+}}{p^{+}} \int d^{2} p_{\perp} \rho_{P}(\mathbf{p})=1 .
$$

With the definitions $z=2 p^{+} / P^{+}$and $\vec{k}_{\perp}=\vec{p}_{\perp}-\frac{1}{2} z \vec{P}_{\perp}$ the density $\rho\left(z, \vec{k}_{\perp}\right)=\rho_{P}(\mathbf{p})$ satisfies

$$
\int_{0}^{2} \frac{d z}{z} \int d^{2} k_{\perp} \rho\left(z, k_{\perp}\right)=1
$$

Conventional nuclear ground-state wave functions are eigenfunctions of a mass operator which are easily related to eigenfunctions of the four-momentum operator. In particular a deuteron wave function which satisfies

$$
\left(V+\vec{k}^{2} / m\right) \varphi_{d}=\varphi_{d} E_{d}
$$

where $V$ is any "realistic" nucleon-nucleon potential, and is an eigenfunction of an invariant two-nucleon mass operator,

$$
\left(4 \omega^{2}+4 m V\right) \varphi_{d}(\vec{k})=\varphi_{d}(\vec{k}) M_{d}^{2}\left(1-E_{d}^{2} / M_{d}^{2}\right),
$$

with $\omega=\sqrt{\vec{k}^{2}+m^{2}}$ and

$$
\begin{aligned}
& \varphi_{d \sigma}\left(\vec{k}, \sigma_{1}, \sigma_{2}\right)=u(|\vec{k}|)\left(\frac{1}{2}, \frac{1}{2}, \sigma_{1}, \sigma_{2} \mid 1, \sigma\right) \\
& +\sum_{m, \sigma^{\prime}} w(|\vec{k}|) Y_{2}^{n}(\hat{k})\left(\frac{1}{2}, \frac{1}{2}, \sigma_{1}, \sigma_{2} \mid 1, \sigma^{\prime}\right)\left(2,1, n, \sigma^{\prime} \mid 1, \sigma\right) .
\end{aligned}
$$

The ratio $E_{d}^{2} / M_{d}^{2} \approx 10^{-6}$ is negligible. The invariant momentum density is

$$
\rho(\vec{k})=\frac{1}{4 \pi}\left(|u(|\vec{k}|)|^{2}+|w(|\vec{k}|)|^{2}\right), \quad \int d^{3} k \rho(\vec{k})=1 .
$$

The density $\rho\left(z, k_{\perp}\right) \equiv \rho\left(z,-k_{\perp}\right) \equiv \rho\left(2-z, k_{\perp}\right)$ is related to $\rho(\vec{k}) \equiv \rho(-\vec{k})$ by

$$
\rho\left(z, k_{\perp}\right)=z \int_{-\infty}^{+\infty} d k_{z} \rho(\vec{k}) \delta\left(z-1-k_{z} / \omega\right) .
$$


With these variable transformations the convolution relation of the structure functions, (18) takes the form

$$
\begin{aligned}
\bar{F}_{2 N}\left(\nu, Q^{2}\right)= & \int_{0}^{2} d z \int d^{2} k_{\perp} \frac{1}{z-\Delta_{N}} \rho\left(z, k_{\perp}\right) \\
& \times F_{2 N}\left(\nu_{N}, Q^{2}\right) \theta\left(m \nu_{N}-\frac{1}{2} Q^{2}\right),
\end{aligned}
$$

with

$$
\Delta_{N}=\frac{k_{\perp} \cdot Q_{\perp}}{m \nu}=\frac{2 \vec{k} \cdot \vec{Q}_{\perp}}{Q^{2}} x, \quad \nu_{N}=\nu\left(z-\Delta_{N}\right) .
$$

The Melosh rotations which relate null-plane spins to canonical spins do not affect these relations. Whether in practice the structure functions are parameterized as functions of $\nu$ and $Q^{2}, x$ and $Q^{2}$, or $\xi$ and $Q^{2}$, is a matter of convenience. For interpolations and averaging of nucleon data, $F_{2}\left(\xi, Q^{2}\right)$ has been used effectively. In the convolution, there is no particular justification for the use of of $\xi$, and $x$ is a more convenient choice. The kinematic constraint on the $\nu$ and $Q^{2}$ dependence of the structure functions implies $0 \leq x \leq 1$ for the nucleons and $0 \leq x \leq 2$ for the deuteron, making it convenient to represent structure functions as functions $F_{2}\left(x, Q^{2}\right)$. In that representation the convolution relation, (26), takes the following form:

$$
\bar{F}_{2 N}\left(x, Q^{2}\right)=\int d^{3} k \frac{z(\vec{k})}{z(\vec{k})-\Delta_{N}} \rho(|\vec{k}|) F_{2 N}\left(x_{N}, Q^{2}\right) \theta\left(1-x_{N}\right),
$$

where

$$
z(\vec{k})=1+k_{z} / \omega, \quad x_{N}=\frac{x}{z-\Delta_{N}} .
$$

Note that in the case of the deuteron, $\vec{k}_{p}=-\vec{k}_{n}$, so $z_{p, n}=1+k_{z}^{p, n} / \omega=1 \pm k_{z}^{p} / \omega$ and $\Delta_{p}=-\Delta_{n}$.

The main contributions are from values of $z$ in the neighborhood of one (between 0.8 and 1.2), and $\Delta_{N}$ is small compared to one, but not negligible. In the limit of $\Delta_{N} \rightarrow 0$, the relation (28) reduces to the familiar deep-inelastic convolution:

$$
\begin{aligned}
\bar{F}_{2 N}\left(x, Q^{2}\right) & =\int d z f(z) F_{2 N}\left(\frac{x}{z}, Q^{2}\right) \theta(z-x), \\
f(z) & =\int d^{2} k_{\perp} \rho\left(z, k_{\perp}\right) .
\end{aligned}
$$

\section{Extraction of $F_{2 n}$ from Proton and Deuteron Data}

\subsection{Extraction procedure}

Given the fit for $F_{2 p}$ from [14, and the deuteron momentum density calculated from the CD-Bonn potential [29], we use (28) to calculate $\bar{F}_{2 p}$. With a parameterization of $F_{2 n}$ we obtain $\bar{F}_{2 n}$ and thus values of $F_{2 d}$, which are compared to the data. Because the ratio $R_{d p}=F_{2 d} / F_{2 p}$ is measured with smaller uncertainties than the absolute structure functions, we compare the calculated values of $R_{d p}$ with the measured values to fit the parameterization of the neutron structure function. We parameterize $R_{n p}$ at $Q^{2}=12 \mathrm{GeV}^{2}$ and then use MINUIT [30] to find the fit to $R_{n p}$ that best reproduces the measured deuteron to proton ratio.

At fixed $Q^{2}$ it is a matter of convenience whether one parameterizes $R_{n p}=$ $F_{2 n} / F_{2 p}$ as a function of $x$ or $\xi$. We chose to parameterize $R_{n p}$ as a function of $\xi$, with 
the expectation that $F_{2 n}\left(\xi, Q^{2}\right)$ will have a smaller $Q^{2}$ dependence than $F_{2 n}\left(x, Q^{2}\right)$, as is the case for the proton and deuteron. We parameterize the $R_{n p}\left(\xi, Q_{0}^{2}\right)$ using the following form,

$$
\begin{aligned}
& R_{n p}(\xi)=\left(p_{1}+p_{2} \xi\right)+p_{3} \exp \left(-p_{4} \xi\right) \\
& +p_{5} \exp \left(-p_{6}(1-\xi)\right)+p_{7}\left[\max \left(0, \xi-p_{8}\right)\right]^{2},
\end{aligned}
$$

where the linear and first exponential terms are the dominant pieces at low $x$, and the quadratic and exponential terms provide flexibility at large $x$. At $x=0, R_{n p} \approx p_{1}+p_{3}$, as the other terms are negligible. The initial fits gave values of $R_{n p}$ consistent with unity at $x=0$, and so we apply the constraint that the ratio should go to one, and take $p_{3}=1-p_{1}$ for all subsequent analysis. The last two terms yield deviation from the linear behavior at large $x$; by including two terms, we allow the possibility of significant cancellation between these terms, yielding flexibility for a modification of the shape at intermediate $x$ values while still reproducing the data at large $x$.

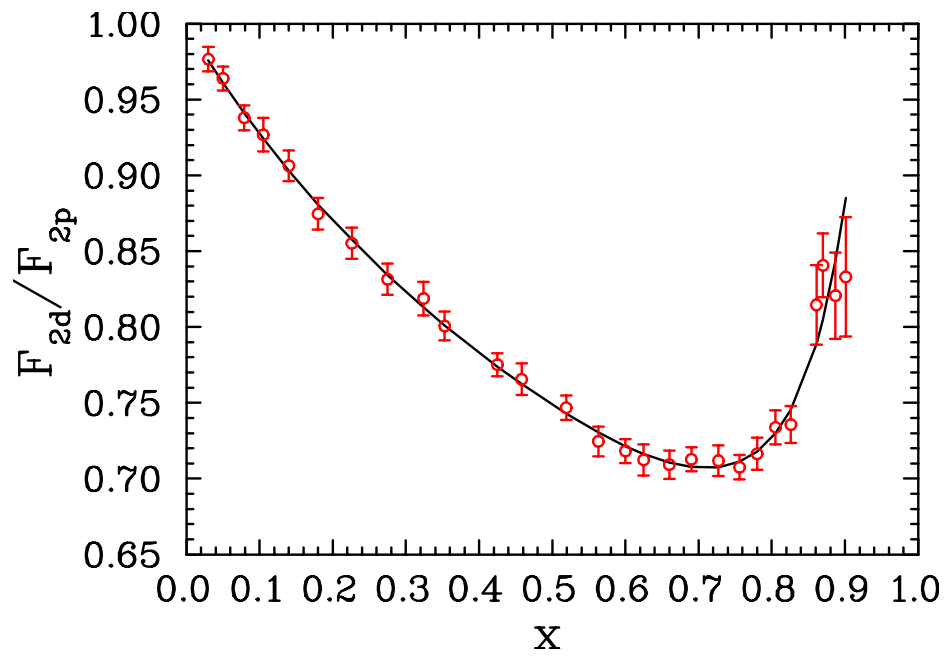

Figure 1. Extracted $F_{2 d} / F_{2 p}$ for $Q^{2}=12 \mathrm{GeV}^{2}$ (points), along with the calculation taking $R_{n p}$ from the best fit using the parameters in table 1

Table 1. Fit parameters for the best fit to $R_{n p}$ using the parameterization from (31). Note that $p_{3}$ is not varied in the fit, and is taken to be $\left(1-p_{1}\right)$.

\begin{tabular}{|c|c|c|c|}
\hline Parameter & value & Parameter & value \\
\hline$p_{1}$ & 0.816 & $p_{5}$ & -0.034 \\
$p_{2}$ & -0.661 & $p_{6}$ & 8.714 \\
$\left(p_{3}\right)$ & 0.184 & $p_{7}$ & -0.072 \\
$p_{4}$ & 5.509 & $p_{8}$ & 0.450 \\
\hline
\end{tabular}

Figure 1 shows the $R_{d p}$ data interpolated to $Q_{0}^{2}=12 \mathrm{GeV}^{2}$. The uncertainties shown are the combined statistical and uncorrelated systematic uncertainties. There is also an estimated 1\% normalization uncertainty [16. The curve in figure 1 shows the calculated $R_{d p}\left(x, Q_{0}^{2}\right)$ for the best fit to $R_{n p}$ (the parameters of the fit are given in table 11). Because the uncertainties are almost evenly split between statistical and correlated systematic except at the largest $x$ values, we expect a reduced chi-squared 


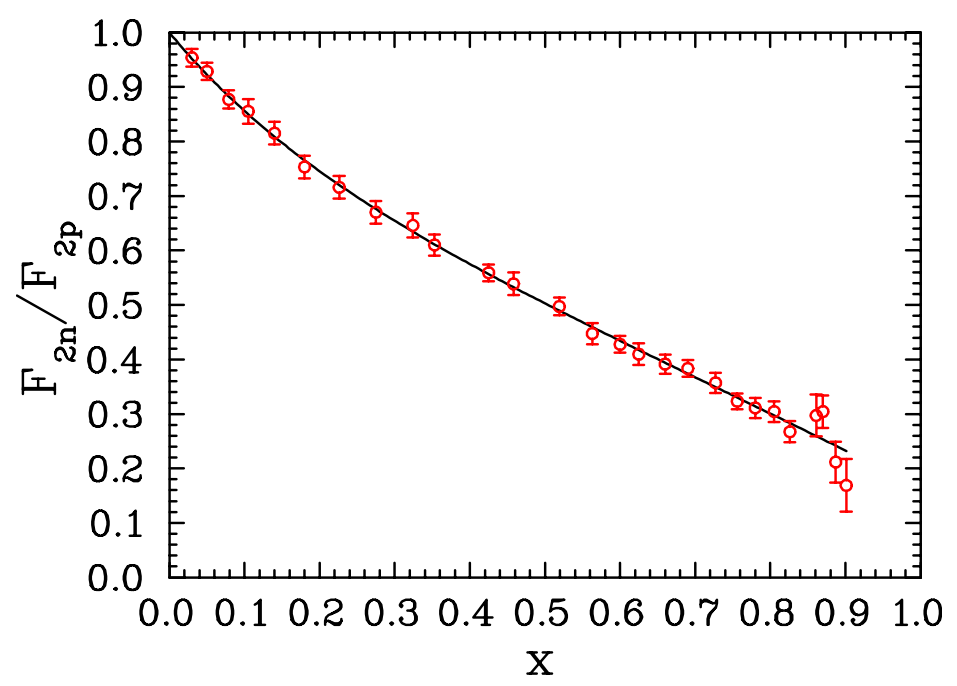

Figure 2. Extracted $F_{2 n} / F_{2 p}$ for $Q^{2}=12 \mathrm{GeV}^{2}$. The data points represent values extracted from $R_{d p}$ measurements according to 33 while the curve is given by (31) with the parameters in table 1

of less then unity. Including the contribution from the normalization paremeters, the fit gives $\chi^{2}=14.6$ for 17 degrees of freedom (27 data points, 7 parameters in the $x$-dependence, and 3 normalization factors).

Figure2 2 shows the fit for $R_{n p}$, along with the values of $R_{n p}$ extracted from the $R_{d p}$ measurements. In terms of the smearing ratios, $S_{N}\left(x, Q^{2}\right)=\bar{F}_{2 N}\left(x, Q^{2}\right) / F_{2 N}\left(x, Q^{2}\right)$, we can write (18) as:

$$
F_{2 d}=\frac{1}{2}\left(S_{p} F_{2 p}+S_{n} F_{2 n}\right),
$$

which allows us to extract $R_{n p}$ using the simple expression:

$$
R_{n p}=\left(2 R_{d p}-S_{p}\right) / S_{n} .
$$

The advantage of extracting individual $R_{n p}$ points using these smearing ratios, calculated from the extracted $F_{2 n}\left(x, Q_{0}^{2}\right)$, is that it provides an estimate of the uncertainty in $R_{n p}$ coming from the uncertainty in the $R_{d p}$ measurements. While this is convenient, and consistent with the way previous extractions have been presented, it does not take into account that a given value of $R_{d p}\left(x, Q^{2}\right)$ depends on the proton and neutron structure functions over a range in $x$. This will be discussed in section 5.2 .

\subsection{Systematic uncertainties}

The extracted $R_{n p}(x)$ values shown in figure 2 include the uncertainties associated with the $R_{d p}$ data, but not the uncertainties associated with the deuteron model and the impulse assumption or systematic uncertainties associated with the extraction procedure. These uncertainties tend to be important at large $x$, and will tend to have a highly correlated impact on the high- $x$ results, and thus the extrapolation to $x=1$. Because the systematic uncertainties were observed to grow with $x$, and yield highly correlated corrections among the high- $x$ points, we make estimates of the uncertainties and then fit these to an exponential, to provide a simple parameterization of the correlated systematic error band. 
The largest systematic at all $x$ values was due to the $1 \%$ overall normalization uncertainty on the global $R_{d p}$ measurements. Shifting the deuterium ratios by $1 \%$ yields an overall absolute shift in $R_{n p}$ of approximately 0.014 , plus an additional contribution at large $x$ values. The large $x$ contribution is well described by $\delta R_{n p}=$ $0.079 \exp (-12(1-x))$.

The result is also sensitive to the parameterization of the proton structure function at large $x$. We vary the high- $x$ behavior of $F_{2 p}\left(x, Q^{2}\right)$, introducing a $10 \%$ change in the falloff at large $x$, which is large enough that the $F_{2 p}$ fit starts to become inconsistent with the SLAC measurements. This yields a change of $\delta R_{n p}=0.06 \exp (-12(1-x))$, where we have used the same $x$ dependence as for the normalization systematic, as the behavior is very similar.

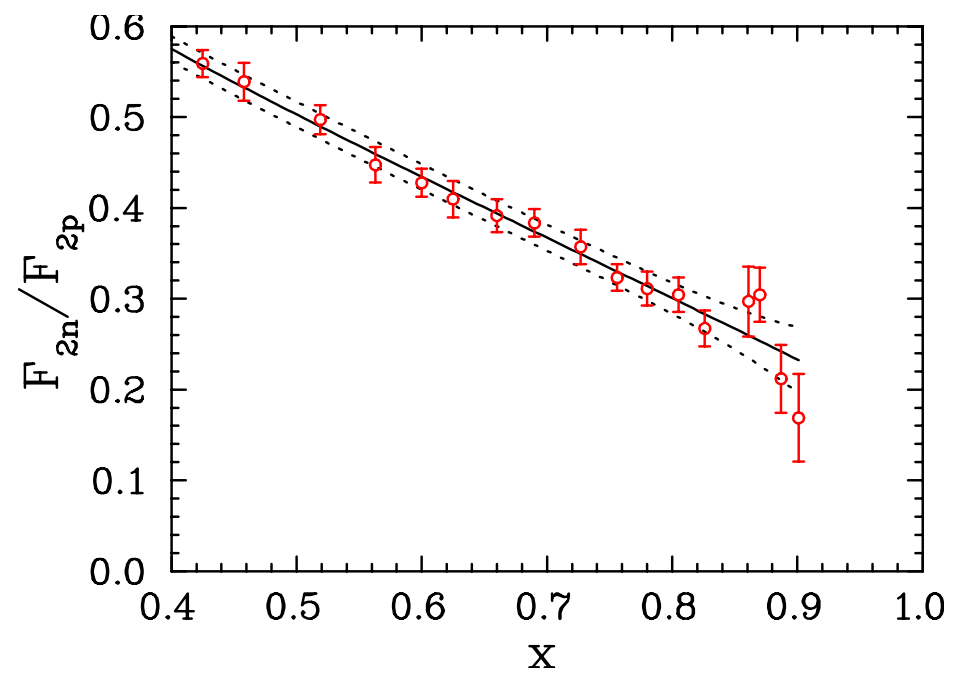

Figure 3. Extracted value of $R_{n p}$, along with the best fit (solid line) and systematic uncertainty bands (dotted lines).

Finally, the uncertainties shown on the $R_{n p}$ data point come only from the statistical and systematic uncertainties on the $R_{d p}$; there is no contribution associated with the uncertainty in the factor $S_{n}$, which depends on the extracted $F_{2 n}$. To account for this, we vary the parameters in the fit to $R_{n p}$ and calculate $R_{d p}$. We compare the extracted $R_{d p}$ to the data, and take the range of fits that increase the total $\chi^{2}$ by one as the range of good $R_{n p}$ fits. For each of these, we recalculate the smearing ratio, $S_{n}$, and determine the impact on the extracted $R_{n p}$ data points. Again, this effect is largest at large $x$, and can be parameterized as $\delta R_{n p}=0.05 \exp (-12(1-x))$.

Combining these systematic uncertainties in quadrature, we find a overall uncertainty of 0.014, and a correlated uncertainty that grows with $x$ of the form $\delta R_{n p}=0.11 \exp (-12(1-x))$. Figure 3 shows the extracted values of $R_{n p}$ at $Q^{2}=12 \mathrm{GeV}^{2}$, along with the error band associated with the systematic uncertainties. Our extracted values, rebinned by a factor of two and including both statistical and systematic uncertainties are given in Table 2 . 
Neutron Structure Functions

Table 2. Extracted values and uncertainties for $R_{d p}(x)$ and $R_{n p}(x)$ at $Q^{2}=12 \mathrm{GeV}^{2}$. Errors labeled "stat" are the combined statistical and uncorrelated systematic uncertainties. Uncertainties labeled "sys" are the correlated systematic uncertainties coming from the relative normalization between different data sets and are correlated. The final column is the uncertainty in the extracion of $R_{n p}$ due to the systematic uncertainties in the extraction procedure discussed in Sec. 5.2 Not included is the overall $1 \%$ normalization uncertainty in $R_{d p}$ which corresponds to an overall shift of \pm 0.014 in $R_{n p}$.

\begin{tabular}{|c||c|c|c||c|c|c|c|}
\hline$x$ & $R_{d p}$ & $\delta R_{d p}^{\text {stat }}$ & $\delta R_{d p}^{\text {sys }} \dagger$ & $R_{n p}$ & $\delta R_{n p}^{\text {stat }}$ & $\delta R_{n p}^{\text {sys }} \ddagger$ & $\delta R_{n p}^{\text {ext }}$ \\
\hline 0.030 & 0.976 & 0.006 & 0.005 & 0.954 & 0.013 & 0.010 & 0.000 \\
0.050 & 0.964 & 0.006 & 0.005 & 0.929 & 0.013 & 0.010 & 0.000 \\
0.079 & 0.938 & 0.006 & 0.005 & 0.877 & 0.012 & 0.011 & 0.000 \\
0.105 & 0.927 & 0.008 & 0.008 & 0.855 & 0.016 & 0.015 & 0.000 \\
0.140 & 0.906 & 0.008 & 0.007 & 0.815 & 0.015 & 0.014 & 0.000 \\
\hline 0.180 & 0.875 & 0.008 & 0.007 & 0.753 & 0.015 & 0.014 & 0.000 \\
0.226 & 0.855 & 0.008 & 0.007 & 0.716 & 0.015 & 0.014 & 0.000 \\
0.275 & 0.831 & 0.008 & 0.007 & 0.670 & 0.015 & 0.014 & 0.000 \\
0.324 & 0.819 & 0.010 & 0.006 & 0.646 & 0.019 & 0.011 & 0.000 \\
0.353 & 0.801 & 0.007 & 0.007 & 0.610 & 0.014 & 0.013 & 0.000 \\
\hline 0.425 & 0.775 & 0.006 & 0.005 & 0.559 & 0.012 & 0.009 & 0.000 \\
0.458 & 0.766 & 0.008 & 0.007 & 0.539 & 0.016 & 0.014 & 0.000 \\
0.519 & 0.747 & 0.006 & 0.005 & 0.497 & 0.012 & 0.010 & 0.000 \\
0.563 & 0.725 & 0.007 & 0.006 & 0.447 & 0.015 & 0.013 & 0.001 \\
0.600 & 0.718 & 0.006 & 0.005 & 0.428 & 0.012 & 0.010 & 0.001 \\
\hline 0.625 & 0.712 & 0.008 & 0.006 & 0.410 & 0.016 & 0.012 & 0.001 \\
0.660 & 0.709 & 0.007 & 0.006 & 0.391 & 0.014 & 0.012 & 0.002 \\
0.690 & 0.713 & 0.006 & 0.005 & 0.383 & 0.012 & 0.009 & 0.003 \\
0.727 & 0.712 & 0.008 & 0.006 & 0.357 & 0.015 & 0.012 & 0.004 \\
0.756 & 0.707 & 0.007 & 0.004 & 0.323 & 0.012 & 0.008 & 0.006 \\
\hline 0.780 & 0.716 & 0.009 & 0.006 & 0.311 & 0.015 & 0.011 & 0.008 \\
0.805 & 0.734 & 0.009 & 0.006 & 0.304 & 0.016 & 0.010 & 0.011 \\
0.826 & 0.736 & 0.011 & 0.005 & 0.267 & 0.018 & 0.009 & 0.014 \\
0.861 & 0.815 & 0.025 & 0.008 & 0.297 & 0.037 & 0.012 & 0.021 \\
0.870 & 0.841 & 0.020 & 0.007 & 0.304 & 0.028 & 0.011 & 0.023 \\
\hline 0.887 & 0.821 & 0.027 & 0.009 & 0.212 & 0.036 & 0.012 & 0.028 \\
0.901 & 0.833 & 0.038 & 0.009 & 0.169 & 0.047 & 0.011 & 0.034 \\
\hline$\dagger$ Additional 1\% scale uncertainty is not shown. & & \\
\hline$\ddagger$ Additional scale uncertainty of 0.014 is not shown. & & \\
\hline
\end{tabular}

\section{3. $Q^{2}$ dependence}

While the analysis is performed at fixed $Q^{2}$, we fit $R_{n p}$ as a function of $\xi$, with the expectation that the $Q^{2}$ dependence will be minimal when taken at fixed $\xi$, as is observed for the proton and deuteron structure functions. By assuming that this parameterization is a reasonable representation of the $Q^{2}$ dependence of the ratio, we can use this and the $Q^{2}$-dependent fit to the proton structure function 14 to examine the $Q^{2}$ dependence of the calculated $R_{d p}$.

Figure 4 shows $R_{d p}$ calculated at $Q^{2}=6$ (bottom curve), 12 , and $20 \mathrm{GeV}^{2}$ (top curve). There is a significant $Q^{2}$ dependence at large $x$, coming mainly from the $Q^{2}$ dependence of $F_{2 p}$. We compare this with the data, now taken in smaller $Q^{2}$ ranges (48, 8-16, and $16-32 \mathrm{GeV}^{2}$ ), interpolated to the same $Q^{2}$ values as the curves. While the data at large $x$ is limited in the $Q^{2}$ range, there is a clear $Q^{2}$ dependence to the measured ratios which is at least in qualitative agreement with the result of the calculation based on the extracted $R_{n p}(\xi)$. 


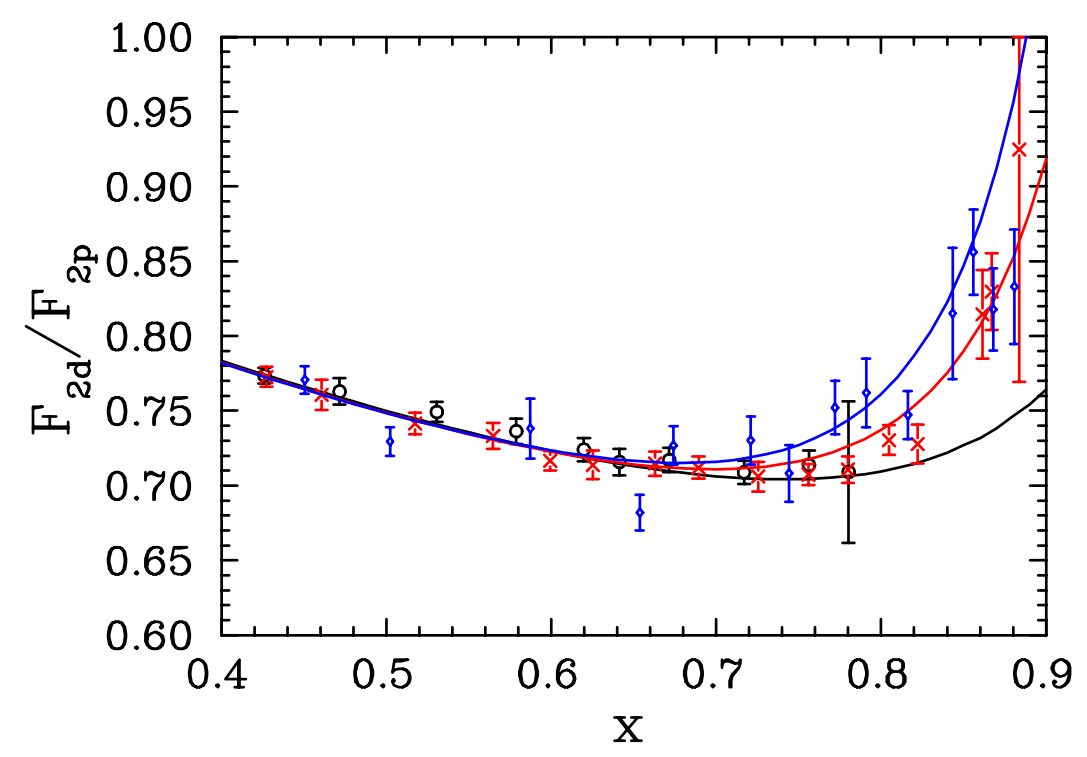

Figure 4. Ratio of deuteron to proton structure function for $Q^{2}$ values from 4-8 $\mathrm{GeV}^{2}$ (black circles), $8-16 \mathrm{GeV}^{2}$ (red crosses), and $16-32 \mathrm{GeV}^{2}$ (blue diamonds), compared with calculations at $Q^{2}=6,12$, and $20 \mathrm{GeV}^{2}$, using the 7parameter fit to $R_{n p}(\xi)$ shown in figure 2

\section{Discussion of the Results}

In the deep-inelastic limit, $x^{2} \ll Q^{2} / 4 m^{2}$, structure functions are related to parton distributions $d(x)$ and $u(x)$. In that limit,

$$
\frac{d\left(x, Q^{2}\right)}{u\left(x, Q^{2}\right)} \approx \frac{4 R_{n p}\left(x, Q^{2}\right)-1}{4-R_{n p}\left(x, Q^{2}\right)},
$$

plus small contributions from heavier quarks. For $Q^{2}=12 \mathrm{GeV}^{2}$ this is expected to be valid for $x \lesssim 0.5$. Our extraction of $R_{n p}(x)$ is consistent with that calculated from the CTEQ6L 31] parton distributions at all $x$ values, although both extractions have large uncertainties at the larger $x$ values, where the simple connection to the quark distributions is expected to break down.

At large $x$, the quantitative features of the extracted neutron structure function are limited by the availability of high precision deuteron and proton data. Existing measurements provide a reasonable basis for the extraction of $R_{n p}$ with high precision up to $x=0.85$, but no significant constraint for $x>0.9$. It follows from the proton and deuteron data, and the deuteron structure assumptions we specified, that the neutron to proton ratio is small for large $x$, although there is little to constraint the extrapolation to $x=1$.

In figure 5 we compare the $R_{n p}$ ratio obtained in section 5 to previously published values of $F_{2 n} / F_{2 p}$. The solid squares are the results of Whitlow et al. [16, who use the DIS convolution and the deuteron wavefunction of the Paris potential [33]. The hollow squares are the results of [32, which emphasizes the off-shell effects of the Buck-Gross [34] spectator representation of the deuteron.

There are a few difficulties in comparing these previous results to our extraction. As mentioned in section 5.2, the previous extractions compared in figure 5 neglect the 


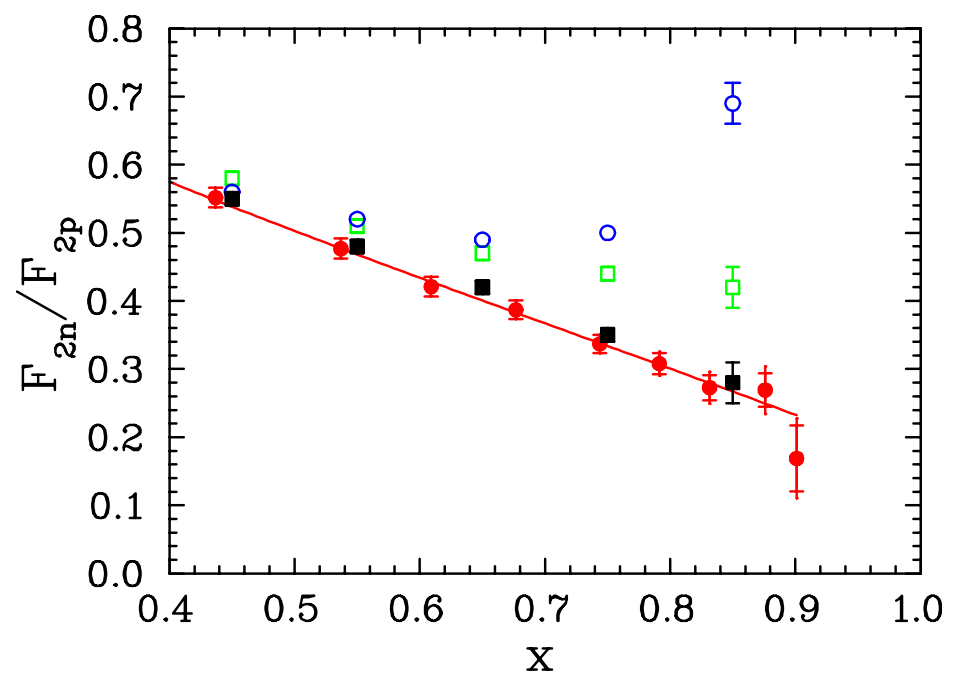

Figure 5. Extracted $F_{2 n} / F_{2 p}$ for $Q^{2}=12 \mathrm{GeV}^{2}$ (solid circles) compared to previous extractions. The hollow squares are points from [32, while the other points are from [16, using a DIS convolution (solid squares) and using a densitydependent extrapolation of the EMC effect (hollow circles).

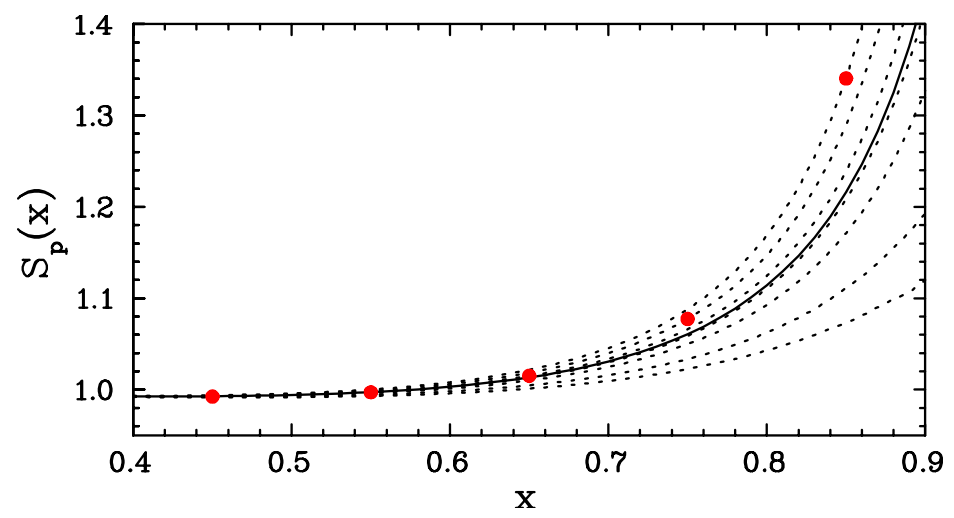

Figure 6. The smearing ratio, $S_{p}$, calculated at several $Q^{2}$ values. The solid line is $12 \mathrm{GeV}^{2}$, while the dotted lines correspond to the $Q^{2}$ values of the $R_{d p}$ points extracted by Whitlow [16, and used in previous analysis. The lowest curve corresponds to $Q^{2}=4.7 \mathrm{GeV}^{2}$, while the upper curve is $23.6 \mathrm{GeV}^{2}$. The points are placed on the curve associated with the average $Q^{2}$ for that $x$ value, which is significantly different from the $12 \mathrm{GeV}^{2}$ value for the large $x$ points.

fact that the uncertainty in the fits to $F_{2 p}$ and the extracted $F_{2 n}$ affect the smearing ratios $S_{p}$ and $S_{n}$ (32), and thus their total uncertainties are close to our statistical uncertainties, as most of the additional systematics are neglected. In addition, the $x$ binning is very coarse. For $x>0.7$, there is a significant $x$-dependence to $R_{d p}$. If the average $x$ of the data in one of the large $x$ bins is shifted from the center of the bin, placing the point at the central $x$ value will affect the results. 
More important is the strong dependence of the smearing ratios on $Q^{2}$, combined with the strong variation of the average $Q^{2}$ value used for each $x$ bin which affects the results compared in figure 5. These extractions used $R_{d p}$ values from [16, where each $x$ value has a different average $Q^{2}$. The low $x$ points have $\left\langle Q^{2}\right\rangle$ values below $8 \mathrm{GeV}^{2}$, while the highest $x$ point has $\left\langle Q^{2}\right\rangle=23.6 \mathrm{GeV}^{2}$. The extraction of 32 calculates $S_{p}$ and $S_{n}$ at $Q^{2}=12 \mathrm{GeV}^{2}$, and uses this for all $x$ values. However, the smearing functions have a strong dependence on $Q^{2}$ at large $x$ values, as shown for $S_{p}$ in Figure 6. For $x=0.85$, the difference between $S_{p}$ at $23.6 \mathrm{GeV}^{2}$ at $12 \mathrm{GeV}^{2}$ (the solid line) is $12 \%$, and so the proton contribution to $R_{d p}$ should be approximately 0.12 higher, making $R_{n p}$ roughly 0.12 lower. A precise comparison of the results obtained using these different models for the deuteron structure contributions will require a self-consistent treatment of the data in the extraction of $R_{n p}$.

If one assumes [35] that the ratio $2 F_{2 d} /\left(F_{2 p}+F_{2 n}\right)$ can be obtained by scaling the EMC ratio, $F_{2 A} / F_{2 d}$, then $R_{n p}$ can be extracted from $F_{2 d} / F_{2 p}$ data without calculating smearing ratios $S_{p}$ and $S_{n}$. Such an extraction was performed in [16, yielding the largest values (hollow circles) for the ratio in figure 5 . This procedure involves the questionable assumption that the details of the nucleon momentum distribution in the deuteron are not important 36, 37. Further, it entirely neglects the important difference between $S_{p}$ and $S_{n}$ by extracting a universal smearing ratio from the extrapolation of the EMC ratio.

No clear conclusions can be drawn from comparisons of the results of different extractions unless they take consistent proton and deuteron data as input and properly treat the $Q^{2}$ dependence of the data. Given proton and deuteron data at fixed $Q^{2}$, the effect of different deuteron structure assumptions should be investigated. It will be important not to confuse different structure assumptions with different representations of the same deuteron structure. Here we assumed that the deuteron state is represented by a vector in the two-nucleon Hilbert space (tensor product of two single-nucleon Hilbert spaces), together with the impulse current tensor. The deuteron wave function which implements these assumptions is an eigenfunction of a mass operator which implements one-pion exchange and two-pion exchange with some parameterization of the short range features which affects the high momentum features of the momentum density $\rho(\vec{k})$. This is implemented differently by different potentials which produce high precision fits to nucleon-nucleon scattering data.

Relations to larger nuclei are important. The impulse assumption with null-plane kinematics applied here to the deuteron is easily applied to to larger nuclei, where eigenfunctions of realistic mass operators exist. Ratios of ${ }^{4} \mathrm{He} /{ }^{2} \mathrm{H}$ will test the the smearing effects on the average nucleon structure functions while ${ }^{3} \mathrm{He} /{ }^{2} \mathrm{H}$ ratios will provide an additional test of both the nuclear effects and the extracted the neutron structure function New measurements of these ratios at large $x$ will be available soon from a recent Jefferson Lab measurement 38.

\section{Conclusion}

The relativistic quantum theory of nuclear structure provides the basis for reliable extraction of neutron structure functions from proton and deuteron structure functions. We extract the neutron structure function using an impulse approximation and an effective two-nucleon mass operator. No deep inelastic approximations or quark structure assumptions are involved. We find that sufficiently accurate interpolations of the proton and deuteron data to fixed $Q^{2}$ are essential, and quantitative comparisons 
to other extraction procedures are not possible until these extractions include a proper treatment of the $Q^{2}$ dependence of the data.

We obtain a precise extraction of the neutron structure function up to $x \approx 0.85$, within the context of our deuteron structure approximation. For $x>0.9$, there are no data to constrain the calculation, and any extrapolation of the results to larger $x$ is unreliable. More data, in particular at large $x$ values, will be essential to extending the extraction.

\section{Acknowledgments}

This work was supported by the U. S. Department of Energy, Office of Nuclear Physics, under contract DE-AC02-06CH11357. The authors would like to thank M. E. Christy for his fits to the proton structure function and for useful discussions of the data at large $x$, and to W. Melnitchouk for useful discussions. We thank R. Machleidt for supplying the CD-Bonn wave functions.

\section{References}

[1] Elliott D. Bloom and Frederick J. Gilman. Phys. Rev. Lett., 25:1140, 1970.

[2] Martin Breidenbach et al. Phys. Rev. Lett., 23:935-939, 1969.

[3] J. D. Bjorken, 1967. Proc. of the Third Int'l. Conf. on Electron and Photon Interactions.

[4] J.D.Bjorken and E.A.Paschos. Phys. Rev., 185:1975, 1969.

[5] Richard P. Feynman. Phys. Rev. Lett., 23:1415-1417, 1969.

[6] P. Amaudruz et al. Phys. Lett., B295:159-168, 1992.

[7] M. Arneodo et al. Nucl. Phys., B487:3-26, 1997.

[8] A. C. Benvenuti et al. Phys. Lett., B223:485, 1989.

[9] J. J. Aubert et al. Nucl. Phys., B293:740, 1987.

[10] L. W. Whitlow, Stephen Rock, A. Bodek, E. M. Riordan, and S. Dasu. Phys. Lett., B250:193198, 1990.

[11] Franz Gross and D. O. Riska. Phys. Rev. C, 36:1928, 1987.

[12] F. Coester and D. O. Riska. Annals Phys., 234:141-161, 1994.

[13] F. Coester. Prog. Part. Nucl. Phys., 29:1-32, 1992.

[14] M. E. Christy. private communication.

[15] J. J. Aubert et al. Nucl. Phys., B259:189, 1985.

[16] L. W. Whitlow, E. M. Riordan, S. Dasu, Stephen Rock, and A. Bodek. Phys. Lett., B282:475482, 1992.

[17] L. H. Tao et al. Z. Phys., C70:387-390, 1996.

[18] V. Tvaskis et al. Phys. Rev. Lett., 98:142301, 2007.

[19] L. W. Whitlow. 1990. Ph.D. Thesis, SLAC-Report-0357.

[20] A. C. Benvenuti et al. Phys. Lett., B237:592, 1990.

[21] J. Arrington, D. Day, B. Filippone, A. F. Lung, et al. Jefferson Lab experiment E02-019.

[22] B. W. Filippone et al. Phys. Rev. C, 45:1582, 1992.

[23] I. Niculescu et al. Phys. Rev. Lett., 85:1186-1189, 2000.

[24] J. Arrington et al. Phys. Rev. C, 64:014602, 2001.

[25] W. Melnitchouk, R. Ent, and C. Keppel. Phys. Rept., 406:127-301, 2005.

[26] J. Arrington, R. Ent, C. E. Keppel, J. Mammei, and I. Niculescu. Phys. Rev. C, 73:035205, 2006.

[27] S. K. Bogner, R. J. Furnstahl, and R. J. Perry. Phys. Rev. C, 75:061001, 2007.

[28] U. Oelfke, P. U. Sauer, and F. Coester. Nucl. Phys., A518:593-616, 1990.

[29] R. Machleidt. Phys. Rev. C, 63:024001, 2001.

[30] F. James and M. Roos. Comput. Phys. Commun., 10:343-367, 1975.

[31] J. Pumplin et al. JHEP, 07:012, 2002.

[32] W. Melnitchouk and Anthony William Thomas. Phys. Lett., B377:11-17, 1996.

[33] M. Lacombe et al. Phys. Lett., B101:139-140, 1981.

[34] W. W. Buck and Franz Gross. Phys. Rev. D, 20:2361, 1979

[35] L. L. Frankfurt and M. I. Strikman. Phys. Rept., 76:215-347, 1981.

[36] W. Melnitchouk, I. R. Afnan, F. Bissey, and A. W. Thomas. Phys. Rev. Lett., 84:5455, 2000. 
[37] Un-Ki Yang and A. Bodek. Phys. Rev. Lett., 84:5456, 2000.

[38] J. Arrington. J. Phys. Conf. Ser., 69:012024, 2007. 